\title{
Improved retrospectively gated in-vivo microCT for simultaneous assessment of lung function and anatomy in mice
}

Christian Dullin ( $\nabla$ christian.dullin@protonmail.com )

Universitätsmedizin Göttingen

Angelika Svetlove

Max-Plank-Institute for Multidisciplinary Sciences

Jana Zschüntzsch

Universitätsmedizin Göttingen

Frauke Alves

Max-Plank-Institute for Multidisciplinary Sciences

Research Article

Keywords:

Posted Date: March 7th, 2022

DOI: https://doi.org/10.21203/rs.3.rs-1399246/v1

License: (c) (i) This work is licensed under a Creative Commons Attribution 4.0 International License.

Read Full License 


\title{
Improved retrospectively gated in-vivo microCT for simultaneous assessment of lung function and anatomy in mice
}

\author{
Christian Dullin ${ }^{1,2,3, *}$, Angelika Svetlove ${ }^{2}$, Jana Zschüntzsch ${ }^{4}$, and Frauke Alves ${ }^{1,2,5,6}$ \\ ${ }^{1}$ Institute for Diagnostic and Interventional Radiology, University Medical Center Goettingen, Germany \\ ${ }^{2}$ Max-Plank-Institute for Multidisciplinary Sciences, Translational Molecular Imaging, Goettingen, Germany \\ ${ }^{3}$ Institute for Diagnostic and Interventional Radiology, University Hospital Heidelberg, Germany \\ ${ }^{4}$ Clinic for Neurology, University Medical Center Goettingen, Germany \\ ${ }^{5}$ Clinic for Haematology and Medical Oncology, University Medical Center Goettingen, Germany \\ ${ }^{6}$ Cluster of Excellence "Multiscale Bioimaging: from Molecular Machines to Networks of Excitable Cells" (MBExC), \\ University of Goettingen, Germany \\ *christian.dullin@protonmail.com
}

\begin{abstract}
Retrospective gating $(R G)$ is a well established technique in preclinical computed tomography (CT) to assess 3D morphology of the lung. In RG additional angular projections are recorded typically by performing multiple rotations. Consequently, the projections are sorted according to the expansion state of the chest and those sets are then reconstructed separately. Thus, the breathing motion artefacts are suppressed at a cost of strongly elevated x-ray dose levels. Here we propose to use the entire raw data to assess lung function in addition to retrospectively gated $3 \mathrm{D}$ reconstruction that visualize anatomical structures of the lung. Using this novel RG based x-ray lung function approach (RG based XLF) on the example of the mdx mouse model of Duchenne muscle dystrophy $(\mathrm{mdx}$ ) we accurately obtained both the 3D anatomical morphology of the lung and the thoracic bones as well as the functional temporal parameters of the lung. Thus, RG-based XLF will remove the necessity for separate acquisition procedures by being able to reproduce comparable lung function results in a single low dose CT scan.
\end{abstract}

\section{Introduction}

Lung diseases continue to present a large burden to public health, especially in industrialized countries and currently even more in correlation to coronavirus (COVID-19) infection. In order to better understand pathomechanisms in lung related diseases as well as to test the efficacy of novel therapies, preclinical studies in animal models are required. These models are typically realized in mice, which due to their small size and rapid breathing rate render in-vivo imaging very challenging. Non-invasive readouts, however, are a major asset in preclinical studies as they allow monitoring disease progression and/or response to therapy in the same animal over time. However, in lung diseases these studies are further hindered by complexity of the diseases that cause changes on anatomical, functional and cellular levels. Thus, a combination of different methods and imaging techniques is required for a comprehensive analysis ${ }^{1}$. Computed tomography (CT) is the method of choice for lung imaging because air inside the lung acts as a natural negative contrast agent, allowing a detailed three dimensional (3D) anatomical characterisation. However, the working principle of CT, reconstructing cross-sections of the specimen by analyzing angular distributed projection images, requires the object/subject/mouse to remain static during data acquisition. Thus, breathing motion - if uncompensated - results in motion artifacts. To circumvent that, different strategies can be used: i) imaging in breath hold, ii) prospective gating and iii) retrospective gating (RG). In clinical CT, investigating compliant patients and using extremely short acquisition times allows for breath-hold imaging. On the other hand, breath-hold imaging in preclinical studies with non-compliant subjects requires intubation and ventilation, the latter interrupted during the acquisition process. Hence, preclinical breath-hold imaging is invasive and in mice does not permit acquisition times much longer than 30s. Thus, multiple studies proposed dividing the acquisition process in several breath hold phases followed by resting times in which the subject is ventilated but the acquisition is paused ${ }^{2}$. This strategy does, of course, prolong the total acquisition for the animal and is solely based on the assumption that position and shape of the lung in each breath-hold phase is identical. In contrast to that, prospective gating uses an external trigger signal to stop the acquisition at unwanted expansion states of the chest ${ }^{3}$. Since respiratory rates of mice are much faster than in humans, even under anesthesia, prospective gating of the CT acquisition process is technically challenging as it would require abrupt starting and stopping of the CT gantry and/or blockage of the 
x-ray tube with a fast enough shutter to avoid additional irradiation of the subject. Therefore, so called retrospective gating (RG) is typically employed in preclinical CT imaging ${ }^{4}$. RG is a technique in which more than the required set of angular projection images is recorded, typically by performing additional rotations. After the acquisition (retrospectively) the phase of the breathing motion is derived from the acquired data and the projections are sorted accordingly into different subsets. These subsets are then reconstructed independently mimicking CT imaging in a static situation. While RG effectively increases the quality of the reconstructed data and can be easily implemented, it requires significantly higher x-ray dose levels than non-gated CT imaging and typically only a fraction of the acquired data is used for the final reconstructions. In addition to the visualization of anatomical changes within the lung, the assessment of the lung function is of great interest. In general, plethysmography is used for pulmonary function testing as it does not require a compliant subject unlike spirometry, the method of choice to assess respiratory function in patients ${ }^{5}$. However, the application of plethysmography in mice is rather difficult and the interpretation of the measurement results is challenging due to commonly overlooked influences of temperature and humidity conditions ${ }^{6}$. In recent years we established x-ray based lung function (XLF) that employs cinematic planar low dose $\mathrm{x}$-ray imaging to determine lung function parameters in mice ${ }^{7}$. We showed that the high sensitivity of XLF allowed monitoring lung function non-invasively over time in different mouse models of allergic airway inflammation ${ }^{8,9}$. Although XLF introduces a comparably low x-ray dose of approximately $6 \mathrm{mGy}$, it requires a further measurement in addition to lung CT imaging used to derive anatomical information, thereby prolonging the time in which the mouse needs to remain under anesthesia. Thus, we present a new approach to quantify lung function in the raw data of retrospective gated lung CT scans that circumvents the need for additional x-ray dose and scanning time by an otherwise subsequently performed XLF acquisition. To illustrate the potential of our novel retropsective gating based x-ray lung function measurement approach ( $\mathrm{rgXLF}$ ) we performed $\operatorname{rgXLF}$ and regular planar XLF in the $m d x$ mouse (C57BL/10ScSn-Dmdmdx/J (B110/mdx)) in comparison to healthy control mice. Mdx mice present the most common and well established mouse model used for studying Duchenne muscular dystrophy (DMD) ${ }^{10}$. DMD is characterised by progressive skeletal muscle degradation, gradual weakening of the diaphragm and auxiliary respiratory muscles ${ }^{11}$. In addition, the skeletal morphology of the thorax/spine is changed over the course of the disease. The patients present with reduction in respiratory function which is recapitulated in the $m d x$ phenotype. By assessing the $m d x$ mouse model with rgXLF we succeeded to accurately reproduce the 3D anatomy of the lung and structure of the thoracic bone as well as to derive functional lung parameters comparable to standard XLF. Moreover, we were for the first time able to put additional projections of the acquired data to use which in the original implementation of RG are simply discarded.

\section{Results}

Cinematic x-ray imaging has already successfully been used to quantify breathing motion. The breathing changes the expansion state and the air content of the lung, which in turn modulates the X-ray attenuation and can therefore be detected as a change in the intensity of the lung region over time ${ }^{8}$.

\section{Principle}

To enable quantification of the lung function the breathing motion needs to be extracted from the acquired projection images. To this end the average brightness in a rectangular region over the lung-diaphragm interface is analyzed as indicated in red in Fig.1D at 4 different angles. Breathing modulates the position of the diaphragm and the expansion state of the chest both resulting in a change in the average x-ray transmission over time $U(\alpha)$ (blue-curve, Fig.1A). However, the angular projection of the mouse anatomy as well as the modulation of the x-ray tube intensity caused by the electronics contribute more strongly to the obtained x-ray attenuation function than the breathing as evidenced by the large baseline of obtained function (blue) in the polar plot Fig.1A. To remove this unwanted effect we exploited the facts that the data should be periodic in $360^{\circ}$ and that the anatomy of the mouse is mostly reflected in the first $K$ frequencies of the Fourier transformation of the signal. However, the insert in Fig.1A shows that the modulation of the intensity of the x-ray tube yielded in a non-2pi-periodic function. We therefore extended the function by adding its mirrored version, which always resulted in a periodic function in $4 p i$. Thus, we reconstructed the background signal by inverse Fourier transformation of the first $K$ frequencies and subtracted the results from the original data, resulting in the red trace Fig.1A, which is also shown in a linear plot in Fig.1B. (Note: for the demonstrated example $K=20$ was used). It can be seen in Fig.1A and B (red curves) that the breathing peaks are well defined. To further suppress potential imprecision in the background correction and the beginning and end of the acquisition, the angular ranges from $0-90^{\circ}$ and $630-720^{\circ}$ are discarded as indicated by the black dashed vertical lines in Fig.1B. The amplitude of the remaining angular range was scaled between 0 and 1 and a level of 0.3 was used to detect breathing events (horizontal black line in Fig.1. Example projection images are illustrated in Fig. $1 \mathrm{C}$ at angles of $0,90,180$ and $270^{\circ}$. The normalized power spectra shown in Fig.1D demonstrate that once the strong contributions of shape of the mouse (blue, beginning of the spectrum) is removed the breathing events and their harmonics (asterisks) can clearly be observed. Moreover, in the filtered spectrum (red) a peak at approximately $470 \mathrm{bpm}$ is visible Fig.1D (§) depicting the heart rate of the mouse Fig.1D. 


\section{X-ray dose measurements}

A commercial x-ray dose measurement system was used to measure the dose length product for several acquisition protocols as summarized in Tab.3. Covering the probe with a $1 \mathrm{~cm}$ layer of pork to mimic scattering processes in the mouse did not affect the readings substantially. Note, that the standard CT acquisition protocol of $17 \mathrm{~s}$, includes ramping up the tube voltage for $2 \mathrm{~s}$ resulting in a total exposure time of $19 \mathrm{~s}$. Therefore, our acquisition protocol for rgXLF using a tube voltage of $90 \mathrm{kV}$, a current of $100 \mu \mathrm{A}$, a field-of-view (FOV) of $20 \times 20 \mathrm{~mm}^{2}$ and an acquisition time of $34 \mathrm{~s}$ results in a dose of approximately $37 \mathrm{mGy}$. Whereas the planar XLF measurement performed with the same parameters apart from the tube current of $40 \mu \mathrm{A}$ and a total acquisition time of $30 \mathrm{~s}$, results in a total $\mathrm{x}$-ray dose of approximately $13 \mathrm{mGy}$.

\section{Parametrization of the breathing pattern}

In order to parameterize the obtained breathing pattern we applied the same strategy described by Khan et al. ${ }^{7}$. In short, a level function at $30 \%$ of the relative $\mathrm{x}$-ray attenuation signal was used to identify single breathing events. The start of the inspiration phase $b_{i}$ was defined as point with the highest curvature prior each breathing peak $p_{i}$. The expiration phase is defined as as descending part of the curve from $p_{i}$ until a value of less that $30 \%$ is reached. Although, several parameters can be calculated for each individual event ${ }^{7}$ in this study we focused on the parameter $k$ of a function $f(t)=I_{0} \exp \left(-k * t^{2}\right)+c$ fitted to the expiration phase and of the heart rate measured in Fourier space. To increase the reliability of the fit, the data of all measured expiration phases was pooled.

\section{Validation of the RG based x-ray based lung function measurements in the $\boldsymbol{m d x}$ mouse model}

To evaluate the accuracy of rgXLF we compared it to the established planar XLF method performed in mdx mice and their wild type controls and by using the same analysis pipeline as described above. Fig.2A and B shows that for both the k-value of the expiration phase as well as the heart rate the application rgXLF and classical XLF resulted in similar results (Pearson correlation coefficient of 0.92 for the $\mathrm{k}$-value and heart rate). Both methods allowed to successfully discriminate $m d x$ from wt mice (Mann-Whitney $\mathrm{U}=0$ for both $\mathrm{k}$-value and heart rate) and revealed an elevated k-value and heart rate in $m d x$. Note, that XLF and rgXLF have been performed subsequently. Due to the variations of the breathing rates of $15 \%$ on average between the measurements a perfect correlation can not be expected.

\section{Retrospectively gated CT reconstruction}

The data derived breathing curves (exemplary shown in Fig.1B) were used to sort the angular projections acquired over $720^{\circ}$ rotation into two bins: i) inspiration and ii) expiration. Since the inspiration phases are very short, only a few projections are recorded. Thus, only the expiration phase was $3 \mathrm{D}$ reconstructed. To this end we used the following scheme. We started with the first projection that demonstrated a value of less than 0.1 in the calculated relative breathing function (see ). From this angle on we generated a full set of equally spaced $\left(0.7^{\circ}\right)$ angular projections over $360^{\circ}$. At each angle we took the projection corresponding to the lowest value in the breathing curve in either the first or the second rotation. If both projections in the first and second rotation had a value below 0.1 the average of both frames was used. In addition, the amount of angles at which no frame was found to have a value of less then 0.1 was reported as a measure of the reliability of the approach. Since we applied a standard filtered back projection algorithm for 3D reconstruction, which requires a set of equally distributed angular projections, only in cases in which the breathing events do not largely overlap between the $1^{\text {st }}$ and $2^{\text {nd }}$ rotations, we achieved reconstructions with a sharper delineation of the lung and the absence of motion artifacts as shown in Fig.3B in contrast to the reconstruction demonstrated in Fig.3A obtained without applying RG. Averaging the two frames at the same angle, if both belong to the expiration phase, reduced the noise level and therefore further improved the image quality. This is for instance of utmost importance if the lung needs to be segmented for subsequent analysis as demonstrated in Fig.3C.

\section{Combined functional and anatomical characterization of the $m d x$ mouse model}

The use of our improved RG approach allows to simultaneously quantify anatomical and functional differences in the $m d x$ mice compared to their wild type controls. In Fig.4 representative cross-sections, lungs segmented in 3D and parts of the isolated breathing events are shown for one wild type control Fig.4A,B and a $m d x$ mouse Fig.4C,D. Already the cross-sections Fig.4A and $\mathrm{C}$ demonstrate that the shape and position of the diaphragm is dramatically different between $m d x$ and control mouse. This is further demonstrated in the 3D renderings of the segmented lungs. In $m d x$ post-caval lung lobe appears enlarged and elongated towards the abdomen. Additionally, the lumen of the airways is increased in the $m d x$ animal. Fig.4B and D. Fig.4E show the traces of the breathing events extracted from the raw-data sets of the CT acquisitions according to the principle described above. Clearly, a more rapid decay (larger k-value) was evidenced in $m d x$ mice (red) compared to healthy controls (blue). In addition, the high frequency modulations of the traces represent the heart beat. 


\section{Discussion}

Here we present an extension of the known retrospective gating (RG) approach for in-vivo lung CT imaging evaluated in a mouse model of Duchenne muscular dystrophy (DMD). We demonstrate that when careful filtering is applied, the average $\mathrm{x}$-ray attenuation at the chest region can be used to quantify differences in the breathing motion. The comparison with our established planar x-ray based lung function measurement (XLF) showed that the same k-values of the expiration phase and similar heart rates were obtained. The application of both methods in a $m d x$ mouse model revealed increased k-values and elevated heart rates under isoflurane anesthesia adjusted to a breathing rate of $0.7 \mathrm{~Hz}$. Since a strong correlation of rgXLF and classical XLF was found, an additional imaging session to measure lung function is not needed anymore and the redundant data of RG can now be used more effectively. The original RG approach described by Bartling et al. is applied to suppress motion artifacts of the lung at the cost of elevated x-ray dose levels ${ }^{4}$. The breathing motion that gets detected in the acquired data set is used to re-bin the data, but to our knowledge no information about the lung function has been previously extracted. RG has been successfully applied in variety of CT imaging studies, however mostly in combination with forced ventilation. Bayat et al., for instance, demonstrated that retrospective gating can be used to perform 4D CT imaging in rats to study the movement of different lung areas in detail, to follow recruitment and local collapse of alveolar spaces and potentially in the future to derive maps of local elasticity ${ }^{12}$. However, most of those studies employ much more than 2 rotations to be able to successfully gate the data, resulting in high x-ray dose levels, which cannot be used in a longitudinal experiment. Our imaging protocol uses much less $\mathrm{x}$-ray dose but with a voxel size of $40 \mathrm{um}$ can not resolve the lung structure of a mouse in detail. Thus, pathological changes can only be detected if they affect the lung on a larger scale. However, our protocol with the dose of approximately $37 \mathrm{mGy}$ in combination with a short total acquisition time of only $34 \mathrm{~s}$ makes longitudinal experiments possible. In future, the derived lung function parameters have the potential to supplement other measurements such as whole body plethysmography and may on its own permit monitoring disease progression or therapy response. The here presented approach of rgXLF requires only to define an area crossing the lung. No additional assumption are made. Therefore, the method can also be applied in a local tomography scanning situation ${ }^{13}$, in which not all projection images show regions of free air around the subject. Moreover, the approach is generally applicable to any CT system, if the acquisition frame rate is sufficiently high to allow sampling of the breathing motion. In our case, an isoflurane anesthesia was adjusted to achieve a breathing frequency of $0.7 \mathrm{~Hz}$, which in combination with the $30 \mathrm{~Hz}$ frame rate of the detector enabled to retrieve a significantly different k-value between $m d x$ mice mimicking DMD and their healthy controls. In addition, adjusting the breathing rate allows to ensure that breathing events do not occur at the same angles of - in our case - an acquisition that consists of two consecutive rotations. The entire pipeline is integrated in our free available $\mathrm{x}$-ray based lung function measurement software that can be downloaded at https://gitlab.com/heimdall32/xLFinal. However, the rgXLF approach has its limitation as the contributions of the underlying shape of the mouse can not be removed completely from the breathing signal. Thus, the rgXLF will most likely show limited sensitivity for features affecting tidal air volume, signs of inflammation or fibrosis etc. However, rgXLF as demonstrated here allows to reliably retrieve temporal parameters such as the k-value, relative inspiration time, heart rate and more. Due to the fact that standard RG always comes at the cost of elevated x-ray dose levels, rgXLF will be limited to preclinical research, but in this perspective allows addressing lung anatomy, shape of the thoracic bone and lung function simultaneously. Thus, we believe that our approach will be a valuable add-on to preclinical lung CT imaging approaches which typically already apply standard RG.

\section{Methods}

\section{in-vivo microCT imaging and retrospective gating}

In-vivo microCT imaging was performed using the QuantumFX device (PerkinElmer) operated with the settings in Tab.1.

\section{X-ray based lung function measurement (XLF)}

For validation of the here presented novel imaging method we performed our established $\mathrm{x}$-ray lung function measurements (XLF) based on planar cinematic x-ray imaging. For this purpose the same in-vivo microCT system was utilized with the following parameters Tab.2.

\section{X-ray dose measurements}

A commercial dose measurement system "Diados" type 11003, SN 0173 (PTW) in combination with a CT adapter "Diados CT adapter", SN: 000607, (PTW) and the sensing element SN: 001031 (PTW) was used.

\section{Reconstruction and segmentation of microCT data sets}

A subset of the acquired angular projections resembling a full rotation of $360^{\circ}$ was reconstructed using the proprietary reconstruction software of the QuantumFX in-vivo microCT system (Perkin Elmer). This resulted in volume data sets with a 
matrix of 512x512x512 voxel and an isotropic voxel size of $40 \mu \mathrm{m}$. In this data lungs were segmented with a simple threshold based segmentation algorithm implemented in Scry v6.0.

\section{MDX mouse model}

Mdx mice (C57BL/ 10ScSn mdx) used for breeding were kindly provided by Ralf Herrmann (University of Essen, Germany). $M d x$ mice (2 male, 2 female mice) and healthy controls [C57BL/ 10ScSn] ( 2 male, 2 female mice, from here on referred to as wt) were used for lung function assessment by XLF and CT imaging. All mice were of an age of 30 weeks at the time of data acquisition. The animals were anaesthetised with approximately 2-3\% isoflurane, airflow of $1 \mathrm{~L} / \mathrm{min}$ of 50:50 mix of air and oxygen for both measurement methods adjusting the breathing rate to about $1.4 \mathrm{~s}$ in between inspiration events.

\section{Ethical statement}

All animal in-vivo procedures were performed in compliance with the guidelines of the European Directive (2010/63/EU) and the German animal ethics regulations and were approved by the local ethics office (Niedersaechsisches Landesamt für Verbraucherschutz und Lebensmittelsicherheit, LAVES, ethics approval 33.9-42502-04-18/2763). Furthermore, the study was carried out in compliance with the ARRIVE guidelines (https://arriveguidelines.org).

\section{Software and Statistics}

Lung function measurement was performed with the custom made software xLFinal (https: //git lab.com/heimdall32/ xLFinal). Plots were generated with matplotlib (https://matplotlib.org). Segmentation and rendering of the lung was done in Scry 6.0 a custom made software by the author. For statistical analysis a welch-T-test implemented in PAST ${ }^{14}$ was used and a p-value of less than 0.05 was considered statistically significant.

\section{References}

1. Chen, D. L. \& Kinahan, P. E. Multimodality molecular imaging of the lung. J. Magn. Reson. Imaging 32, 1409-1420, DOI: $10.1002 / j m r i .22385$ (2010).

2. Lovric, G. et al. Tomographic in vivo microscopy for the study of lung physiology at the alveolar level. Sci. Reports 7, 12545, DOI: 10.1038/s41598-017-12886-3 (2017).

3. Shuman, W. P. et al. Prospective versus Retrospective ECG Gating for 64-Detector CT of the Coronary Arteries: Comparison of Image Quality and Patient Radiation Dose. Radiology 248, 431-437, DOI: 10.1148/radiol.2482072192 (2008).

4. Bartling, S. H. et al. Retrospective Motion Gating in Small Animal CT of Mice and Rats:. Investig. Radiol. 42, 704-714, DOI: 10.1097/RLI.0b013e318070dcad (2007).

5. Nensa, F., Marek, W., Marek, E., Smith, H. \& Kohlhäufl, M. Assessment of airway hyperreactivity: comparison of forced spirometry and body plethysmography for methacholine challenge tests. Eur. J. Med. Res. 14, 170, DOI: 10.1186/2047-783X-14-S4-170 (2009).

6. Hülsmann, S. et al. Evaluation of a mechanical lung model to test small animal whole body plethysmography. Sci. Reports 11, 17099, DOI: 10.1038/s41598-021-96355-y (2021).

7. Khan, A. et al. Simple low dose radiography allows precise lung volume assessment in mice. Sci. Reports 11, 4163, DOI: 10.1038/s41598-021-83319-5 (2021).

8. Dullin, C. et al. X-Ray based Lung Function measurement-a sensitive technique to quantify lung function in allergic airway inflammation mouse models. Sci. Reports 6, 36297, DOI: 10.1038/srep36297 (2016).

9. Markus, M. A. et al. X-ray-based lung function measurement reveals persistent loss of lung tissue elasticity in mice recovered from allergic airway inflammation. Am. J. Physiol. Cell. Mol. Physiol. 313, L763-L771, DOI: 10.1152/ajplung. 00136.2017 (2017).

10. McGreevy, J. W., Hakim, C. H., McIntosh, M. A. \& Duan, D. Animal models of Duchenne muscular dystrophy: from basic mechanisms to gene therapy. Dis. Model. \& Mech. 8, 195-213, DOI: 10.1242/dmm.018424 (2015).

11. Grounds, M. D., Radley, H. G., Lynch, G. S., Nagaraju, K. \& De Luca, A. Towards developing standard operating procedures for pre-clinical testing in the mdx mouse model of Duchenne muscular dystrophy. Neurobiol. Dis. 31, 1-19, DOI: 10.1016/j.nbd.2008.03.008 (2008).

12. Bayat, S., Porra, L., Suortti, P. \& Thomlinson, W. Functional lung imaging with synchrotron radiation: Methods and preclinical applications. Phys. Medica 79, 22-35, DOI: 10.1016/j.ejmp.2020.10.001 (2020). 
13. Faridani, A., Ritman, E. L. \& Smith, K. T. Local Tomography. SIAM J. on Appl. Math. 52, 459-484, DOI: 10.1137/0152026 (1992).

14. Hammer, Ø., Harper, D. A., Ryan, P. D. et al. Past: Paleontological statistics software package for education and data analysis. Palaeontol. electronica 4, 9 (2001).

\section{Acknowledgements}

The authors thank Sarah Garbode, Regine Kruse and Bärbel Heidrich for excellent technical assistance in imaging the mice and in supporting data analysis. In addition, we thank Heiko Seeger from the Department of Medical Technologies of the University Medical Center Goettingen for assisting the dose measurements. University Medical Center Goettingen holds a membership of the European Reference Network for Rare Neuromuscular Diseases (ERN EURO-NMD). This work was supported by the Deutsche Forschungsgemeinschaft (DFG, German Research Foundation under Germany's Excellence Strategy - EXC 2067/1390729940 (FA))

\section{Author contributions statement}

C.D., F.A., J.Z. conceived the experiment(s), C.D. and A.S. conducted the experiment(s), C.D. analysed the results. All authors reviewed the manuscript.

\section{Data Availability}

The datasets generated and/or analysed during the current study are available in the open science framework (OSF) repository, DOI 10.17605/OSF.IO/3QPXZ. The generated analysis software can be found here https: / / git lab.com/heimdall 32 / xLFinal;

\section{Additional information}

Competing interests The author(s) declare no competing interests. 

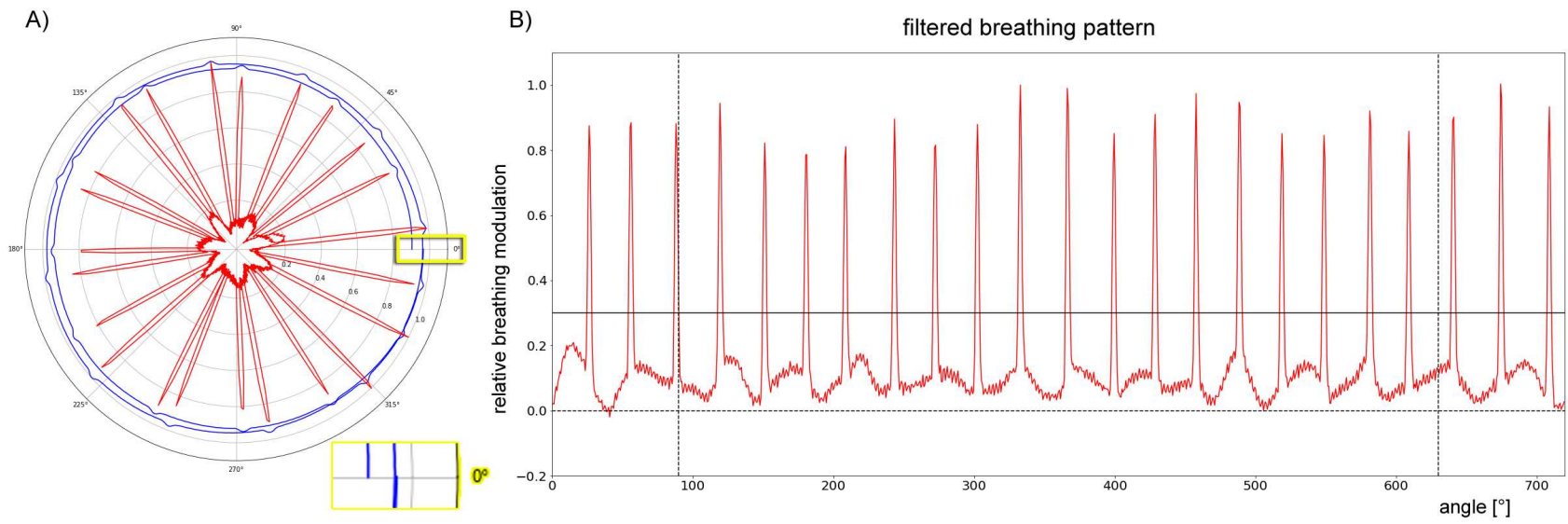

C)
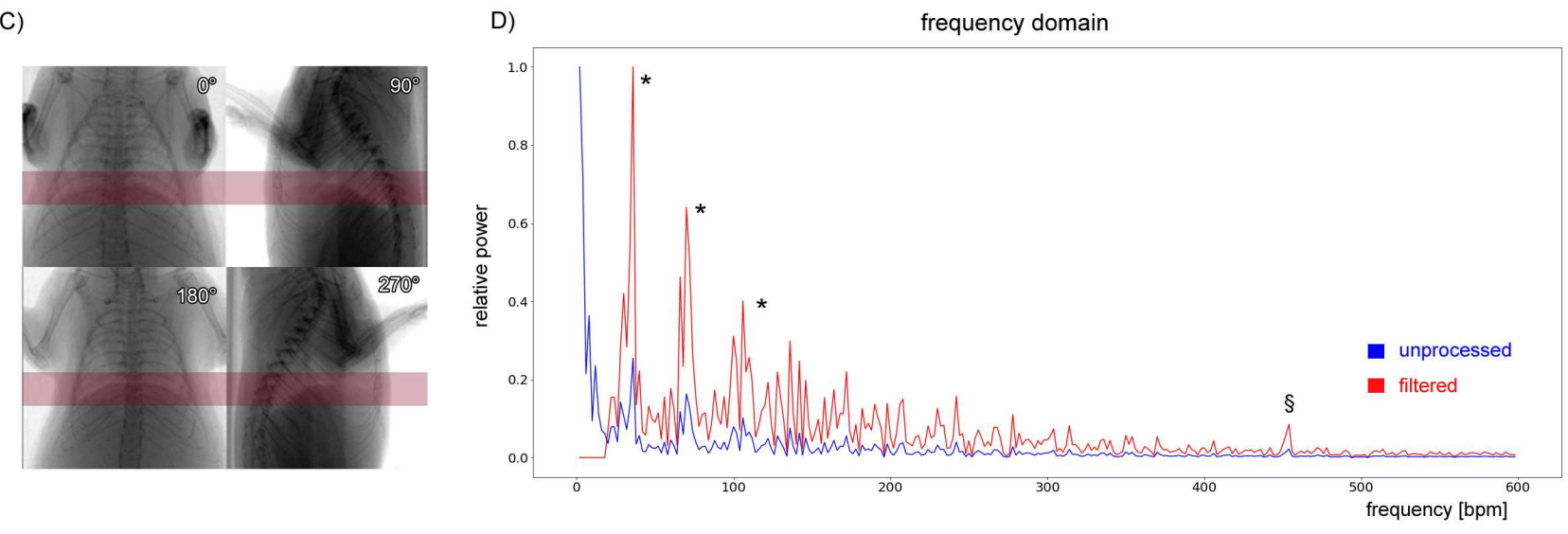

Figure 1. Principle of retrieving the breathing motion from the set of angular distributed projection images. A) Polar plot of the average $\mathrm{x}$-ray transmission over a region of interest (ROI) covering the entire cross-section of the mouse at the lung-diaphragm interface (blue) is shown. The breathing is only visible as subtle modulation of the signal and therefore a background correction is need, which is hindered by the fact that the signal is not strictly 2 pi periodic (insert). The final breathing pattern after background correction is shown in red. To suppress potential imprecision in the background correction and the beginning and end of the acquisition a range of $90^{\circ}$ from the start and end of the measurement (vertical dashed lines) was discarded. The amplitude of the remaining angular range was normalized to [0,1]. A level of 0.3 (horizontal dashed line) was used to identify breathing events in the resulting function. C) Representative projection images are shown at $0,90,180$ and $270^{\circ}$ with the ROI indicated in red. D) Comparison of the power spectra of the unprocessed data (blue) and the processed data (red) shows that after background removal the breathing events and their harmonics can clearly be seen (* asterisks). Moreover, the frequency of the heart beat can be detected as well $(\S)$. 
A)

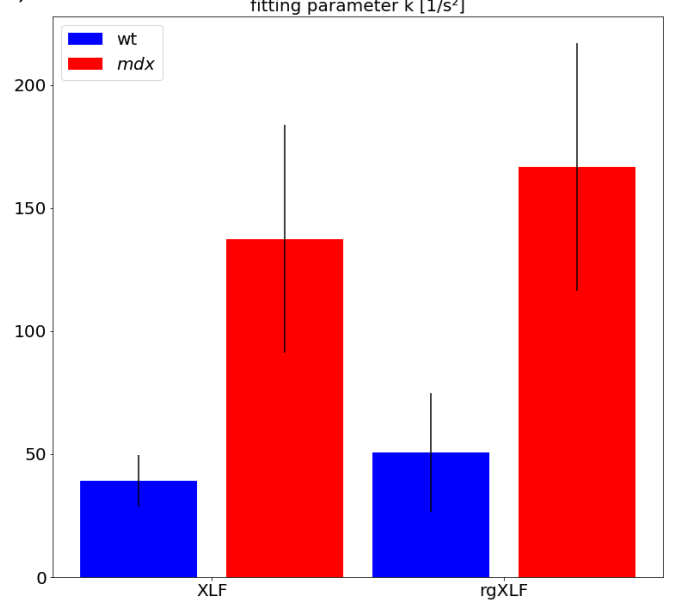

B)

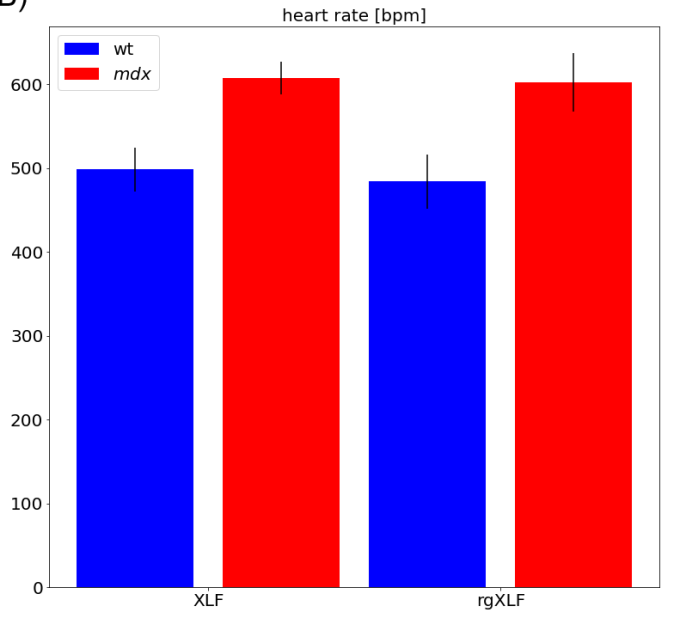

Figure 2. Comparison of the novel RG based lung function measurement (rgXLF) with the established planar XLF. A) shows the calculated $\mathrm{k}$-values for the expiration phase in $m d x$ and wt mice, revealing increased k-values in $m d x$ mice. B) The calculated heart rates for $m d x$ and wt mice demonstrate a faster rate for $m d x$ mice than in wt mice at the same level of anesthesia. Both XLF and rgXLF show similar outcomes that correlate with a Pearson correlation coefficient of 0.92 in both parameters. 
A)

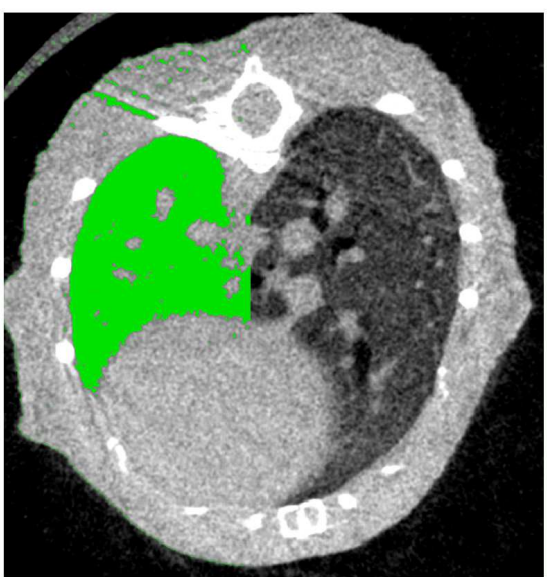

B)

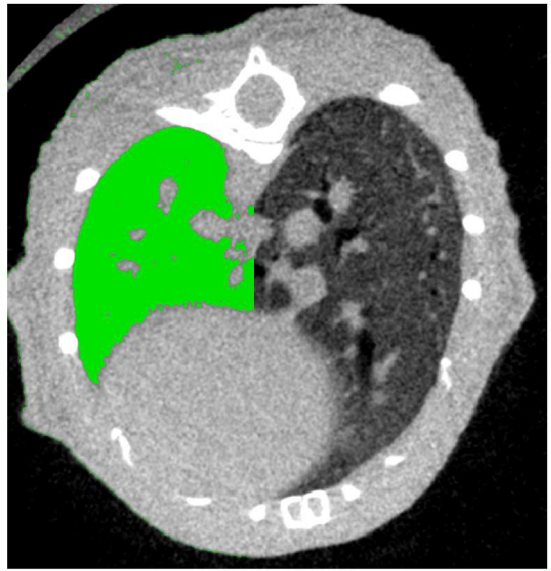

C)

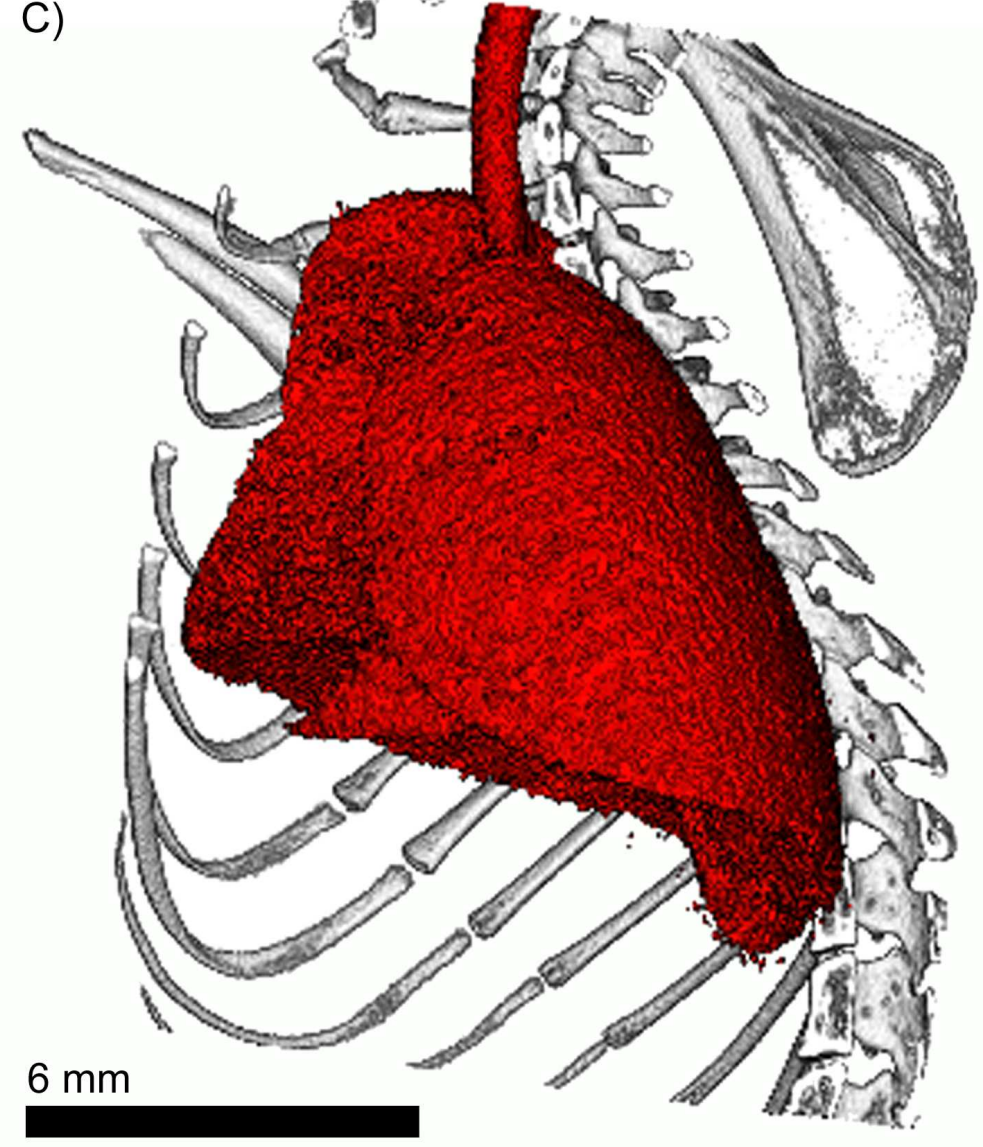

Figure 3. Representative cross section of CT data sets of the chest region of a mouse reconstructed A) without RG and B) with RG and frame averaging. In green the segmented lung using the same threshold is partially overlaid. Clearly, in B) both, less motion artifacts and a sharper delineation of the lung towards the rib cage are observed. This allows to study the anatomical shape of the lung in 3D as demonstrated in C). Note, that the interface between lung and heart was not improved since no gating was performed for the movement of the heart. 

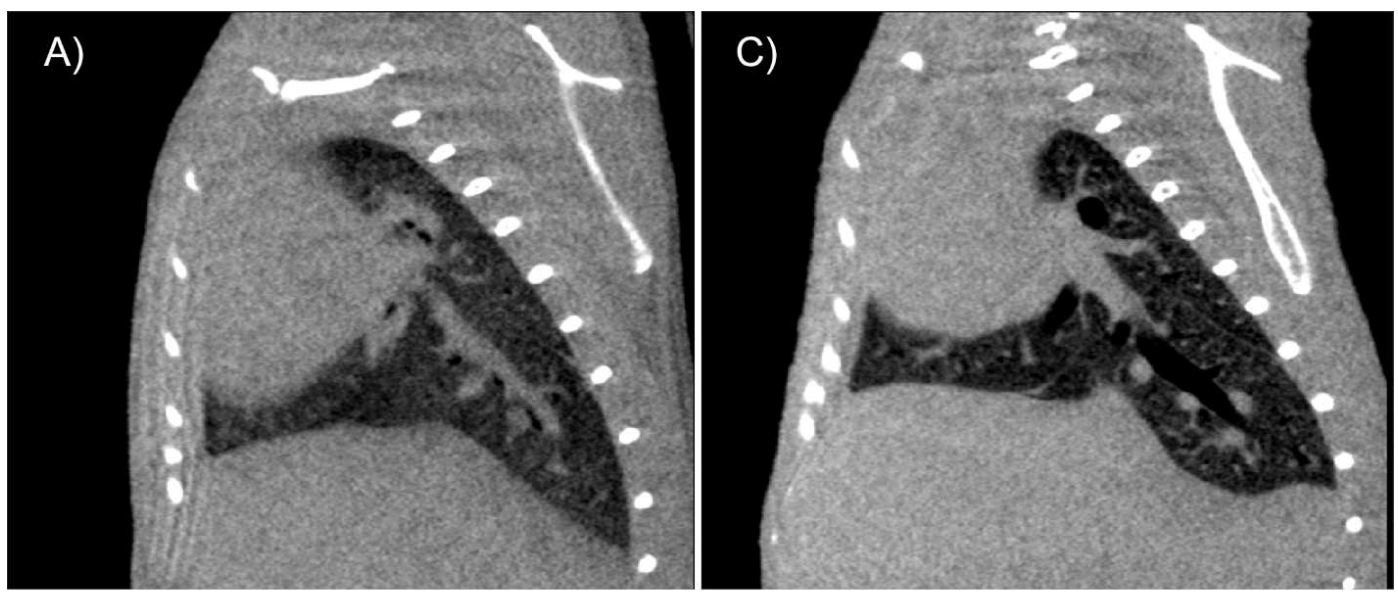

B)

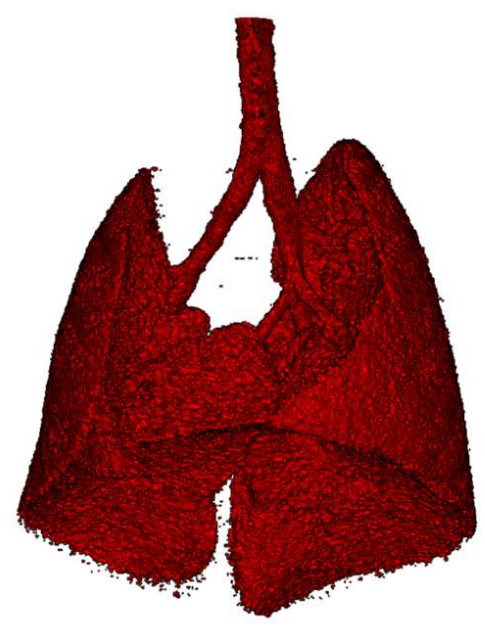

D)

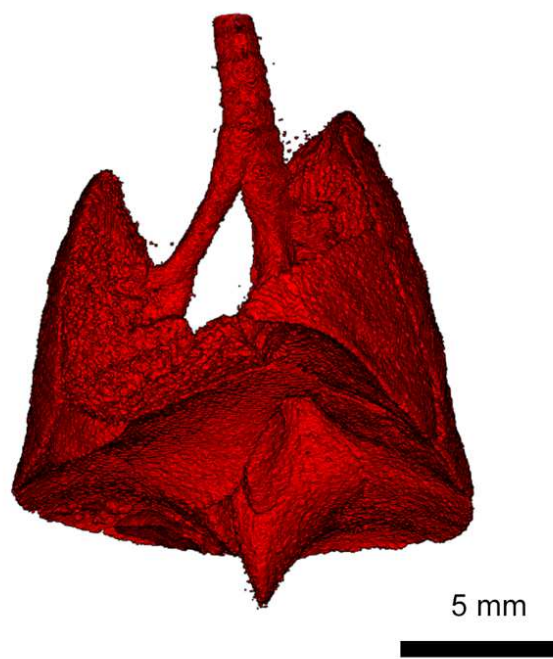

E)

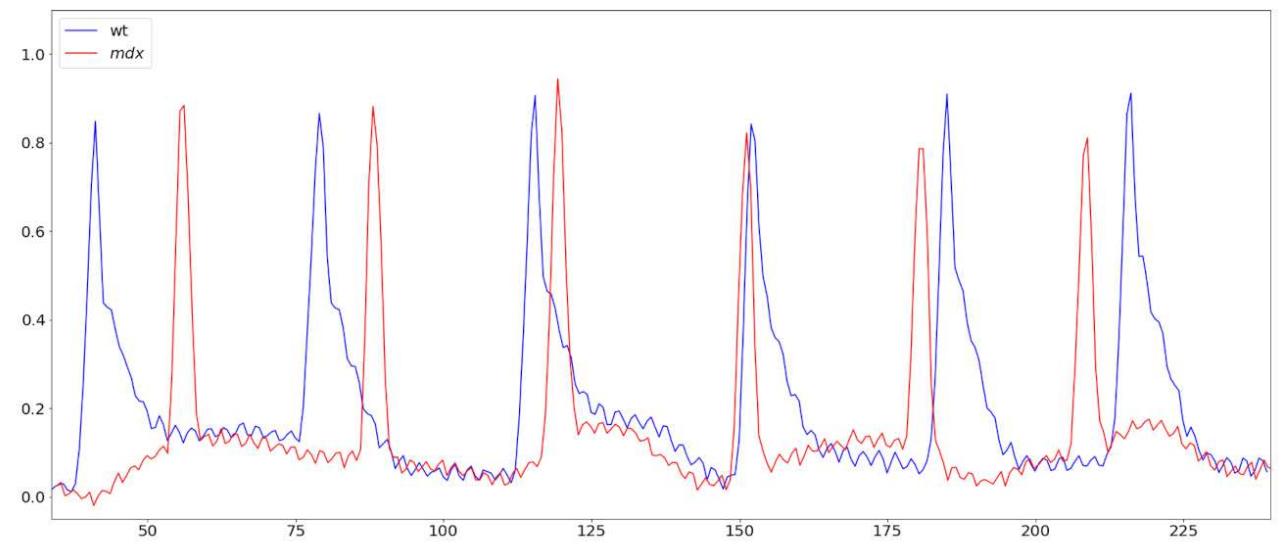

Figure 4. Cross sections of A a CT image of a healthy control in comparison to $\mathrm{C}$ of a $m d x$ mouse show a modified shape and an altered position of the diaphragm in $m d x$. The same deformation of the lung can be observed in the 3D renderings of the segmented lung of the healthy mouse B and the $m d x$ mouse D, especially for the post-caval lobe (§). E shows the extracted breathing pattern for the same mice (healthy=blue and $m d x=$ red). Clearly the $m d x$ mouse displays a more rapid decay in the expiration phase. 


\begin{tabular}{r|r} 
tube voltage & $90 \mathrm{kV}$ \\
\hline tube current & $100 \mu \mathrm{A}$ \\
\hline field of view (FOV) & $20 \times 20 \mathrm{~mm}^{2}$ \\
\hline total acquisition time & $34 \mathrm{~s}$ \\
\hline rotation angle & $720^{\circ}$ \\
\hline total amount of projection images & 1028
\end{tabular}

Table 1. microCT acquisition parameters

\begin{tabular}{r|r} 
tube voltage & $90 \mathrm{kV}$ \\
\hline tube current & $40 \mu \mathrm{A}$ \\
\hline field of view (FOV) & $20 \times 20 \mathrm{~mm}^{2}$ \\
\hline total acquisition time & $34 \mathrm{~s}$ \\
\hline rotation angle & $0^{\circ}$ \\
\hline total amount of projection images & 1024
\end{tabular}

Table 2. parameter for $\mathrm{x}$-ray based lung function measurement

\begin{tabular}{r|r|r|r|r|r} 
covered & voltage $[\mathrm{kV}]$ & current $[\mu \mathrm{A}]$ & FOV $\left[\mathrm{mm}^{2}\right]$ & time $[\mathrm{s}]$ & dose $[\mathrm{mGy}]$ \\
\hline- & 90 & 200 & $73 \times 73$ & 19 & 14.0 \\
- & 90 & 200 & $73 \times 73$ & 19 & 13.9 \\
- & 90 & 200 & $73 \times 73$ & 19 & 14.8 \\
- & 90 & 200 & $40 \times 40$ & 19 & 21.3 \\
- & 90 & 200 & $20 \times 20$ & 19 & 41.6 \\
$\mathrm{x}$ & 90 & 200 & $73 \times 73$ & 19 & 13.8 \\
$\mathrm{x}$ & 90 & 200 & $40 \times 40$ & 19 & 20.0
\end{tabular}

Table 3. dose measurements for different settings. Measurements taken with covering the probe with meat are indicated by ' $x$ ' 